\title{
Size perception biases are temporally stable and vary consistently between visual field meridians
}

Dietrich Samuel Schwarzkopf ${ }^{* 1,2}$

1. Experimental Psychology, University College London, United Kingdom

2. School of Optometry \& Vision Science, University of Auckland, New Zealand

* To whom correspondence should be directed: s.schwarzkopf@auckland.ac.nz

\section{Abstract}

The apparent size of visual stimuli depends on where in the visual field they appear. I recently presented a model of how size perception could be biased by stimulus encoding in retinotopic cortex. However, it remains unclear if such biases are related to general discrimination ability and if they are temporally stable. Moreover, an independent test of the model assumptions is still outstanding. Here I present two experiments to address these issues. Experiment 1 shows that perceptual biases are stable across stimulus durations between 50-1000 ms, even though discrimination ability increases with stimulus duration. Experiment 2 demonstrates that perceptual biases are stronger along the vertical than the horizontal meridian, which mirrors recently reported differences in positional tuning. Taken together, these findings support the predictions of our model of how size is inferred from visual cortical responses. 


\section{Introduction}

Our impression of a seamless and accurate perception across our visual field belies the fact that the neural representation of the visual field is highly heterogeneous. Spatial visual acuity is highest in central vision and falls off with increasing eccentricity meaning that the visual system encodes only coarse spatial detail in the periphery (Anstis, 1998; Dumoulin \& Wandell, 2008). Similarly, visual ability has also been shown to vary considerably between visual field meridians (Anderson, Leslie Cameron, \& Levine, 2014; Carrasco, Talgar, \& Cameron, 2001). More recently, it was shown that for many visual functions there are unique spatial patterns that are reliable but very idiosyncratic in each individual (Afraz, Pashkam, \& Cavanagh, 2010; Greenwood, Szinte, Sayim, \& Cavanagh, 2017; Kosovicheva \& Whitney, 2017; Moutsiana et al., 2016; Schwarzkopf \& Rees, 2013; Visconti di Oleggio Castello, Taylor, Cavanagh, \& Gobbini, 2018).

I recently formulated a model of how visual object size could be read out from the retinotopic representation of stimuli in early visual areas (Moutsiana et al., 2016). This model was derived from the finding that the apparent size of stimuli decreases with eccentricity (Anstis, 1998; Newsome, 1972), and thus broader position tuning in visual cortex (Dumoulin \& Wandell, 2008; Moutsiana et al., 2016; Smith, Singh, Williams, \& Greenlee, 2001) as measured by the spread of population receptive fields ( $\mathrm{pRF}$ ). It also explained why idiosyncratic spatial patterns in the position tuning correlate with the spatial heterogeneity of size perception biases across the visual field (Moutsiana et al., 2016). However, a critical test of this model would require that size perception also varies predictably in independent data when positional tuning is known to be broader. Moreover, it remains unclear in how far these perceptual biases could merely be a consequence of discrimination performance or limited information.

Here, I present two experiments to address these issues. First, I tested if the spatial heterogeneity of size perception biases depends on stimulus 
duration. Using the same paradigm I used in previous studies, participants performed the Multiple Alternatives Perceptual Search (MAPS) task to measure biases in size perception at four parafoveal locations (Finlayson, Manser-Smith, Balraj, de Haas, \& Schwarzkopf, 2018; Finlayson, Papageorgiou, \& Schwarzkopf, 2017; Moutsiana et al., 2016). This entails finding which of the four candidate stimuli perceptually matches the size of a constant reference stimulus shown at fixation. Unlike in previous studies in which the stimuli were only shown for a brief $200 \mathrm{~ms}$, I varied the stimulus duration from $50-1000 \mathrm{~ms}$ and quantified whether or not perceptual biases change with duration.

In a second experiment, I then further tested our prediction that stimuli are perceived as smaller in locations where the positional tuning of neuronal populations in corresponding parts of visual cortex is broader (Moutsiana et al., 2016). A recent study (Silva et al., 2017) suggests that positional tuning is broader (that is, pRF spread is greater) along the vertical than the horizontal meridian, which ties in with previous research suggesting more precise visual perceptual function along the horizontal meridian (Anderson et al., 2014; Carrasco et al., 2001). Considering the link I posited between pRF spread and size perception, I therefore predicted that visual objects should be perceived as smaller (more biased) along the vertical than the horizontal meridian.

\section{Methods}

\section{Participants}

Twenty-one observers (ages 19-38, 13 female, 4 left-handed) participated in Experiment 1, including the author. The experimental protocol of Experiment 2 was preregistered (osf.io/8u2z5). Thirteen observers (ages 20-39, 8 female, 2 left-handed, 1 ambidextrous) participated in Experiment 2, using a Bayesian sampling plan (see below for details). The author also 
took part in this experiment but his results were excluded from the inferential statistical analysis because his data were acquired before preregistration. All observers gave written informed consent and procedures were approved by the University College London Research Ethics Committee. All observers had normal or corrected-to-normal visual acuity. In Experiment 2, I had a predefined exclusion criterion that any observer whose accuracy on the MAPS task for an experimental run was $30 \%$ or less would be excluded (note that chance performance is $25 \%$ ). All observers performed the task above criterion on all runs and therefore nobody was excluded.

\section{Stimuli}

Observers were presented with a stimulus array containing four light grey, parafoveally presented circle stimuli (the candidates) and one reference circle shown in the center of gaze. The background was black. A blue fixation dot $\left(0.2^{\circ}\right.$ visual angle $)$ was also present in the center of gaze. The sizes of three of the candidates relative to the size of the reference $\left(0.98^{\circ}\right.$ visual angle) were drawn from a Gaussian distribution $(\mu=0, \sigma=0.3)$ expressed in binary logarithmic units. The size of the fourth candidate was identical to the size of the reference. These stimuli have been described in more detail previously (Finlayson et al., 2018, 2017; Moutsiana et al., 2016).

In Experiment 1, the candidates were presented along the oblique axis in each visual field quadrant at an eccentricity of $3.92^{\circ}$. Stimulus duration was either $50 \mathrm{~ms}, 100 \mathrm{~ms}, 200 \mathrm{~ms}, 500 \mathrm{~ms}$, or $1000 \mathrm{~ms}$, and these durations were pseudo-randomly interleaved across trials.

In Experiment 2, candidates were presented along the vertical and horizontal meridians at an eccentricity of $3.92^{\circ}$ or $7.84^{\circ}$, the middle and outer eccentricity I had previously used (Moutsiana et al., 2016). Please 
note that in the preregistration document, this was incorrectly defined as $7.94^{\circ}$. Stimulus duration was always fixed at $200 \mathrm{~ms}$.

\section{Procedure}

In both experiments, observers were instructed to fixate a central dot and perform the MAPS task (Finlayson et al., 2018, 2017; Moutsiana et al., 2016). Observers were instructed to select the candidate that appeared most similar in size to the reference using keys on a keyboard corresponding to the four locations. Following their choice, a ripple effect indicated the chosen location and the fixation dot briefly changed by increasing its size to $0.33^{\circ}$ for $50 \mathrm{~ms}$. No feedback about the correctness of the response was given, which differs from most of our previous experiments using the MAPS task. I recently showed that perceptual bias estimates are greater without feedback even though spatial patterns of bases are similar irrespective of whether feedback is given (Finlayson et al., 2018). However, in both experiments most participants were given the opportunity to briefly familiarize themselves with the task before the actual experiment commenced. During these practice trials, feedback was given by turning the fixation dot green for $50 \mathrm{~ms}$ if they had picked the correct target on a trial.

In Experiment 1, the observers' eye movements were recorded at $60 \mathrm{~Hz}$ using a custom desk-based eye tracker which was calibrated prior to the experiment. There were normally 1000 trials in total in Experiment 1, 200 trials per stimulus duration. Every 20 trials, observers were given a brief rest break and asked to continue by pressing any key on the keyboard. Already acquired data were saved at each rest block. Due to an unresolvable technical issue with the eye tracking code, the protocol sometimes crashed. When that happened, the experiment was restarted, and the number of still required blocks reduced accordingly. This meant that some participants performed a small number of additional trials which 
were not recorded. As in previous experiments (Finlayson et al., 2018, 2017; Moutsiana et al., 2016), the keys for making the behavioral response on each trial were $\mathrm{F}, \mathrm{V}, \mathrm{M}$, and $\mathrm{K}$ corresponding to the four visual field quadrants.

In Experiment 2, there were 400 trials per run and observers performed two runs, one for each eccentricity. The order of eccentricity conditions was pseudo-randomized for each observer. As in Experiment 1, there was a rest break every 20 trials. No eye tracking was used in this experiment. The keys for making behavioral responses were the four arrow keys denoting the candidate above, below, left, or right of fixation, respectively.

\section{Data analysis}

I fit a model to the behavioral responses to quantify the perceptual bias, the size required to perceptually match the candidate at a given location to the reference, and the discrimination ability, the uncertainty with which they chose a candidate in a particular location. The model essentially maximizes the prediction of the observer's behavioral response across trials by altering the parameters of a similarity detection function at each candidate location. This procedure has previously been described in much greater detail (Finlayson et al., 2018, 2017; Moutsiana et al., 2016).

In Experiment 1, I quantified how perceptual biases and discrimination performance depended on stimulus duration. For that, I averaged parameter estimates across the four candidate locations because here I was only interested in the magnitude of these measures rather than their spatial patterns. I also quantified the eye position in each trial and analyzed this separately for each duration condition. I removed artifacts by deleting empty samples and any samples for which the horizontal or vertical eye position was further than 3 standard deviations from the mean. I then calculated the variance for the horizontal and vertical positions and then converted this into a Euclidean distance (square root of the sum of the 
squares of these variances). Finally, I removed participants for whom this measure exceeded $4^{\circ}$ visual angle as these constituted excessively noisy recordings.

In Experiment 2, I compared perceptual biases and discrimination performance between the vertical and horizontal meridian. For our main analysis, I averaged parameter estimates across all locations on each meridian irrespective of eccentricity or visual hemifield (i.e., upper and lower hemifield for vertical meridian, left and right hemifield for horizontal meridian). I collected data from 10 observers and then continued sampling until a Bayesian paired t-test (Rouder, Speckman, Sun, Morey, \& Iverson, 2009) comparing perceptual biases for the vertical and horizontal meridians favored either the alternative or the null hypothesis. The default prior had a scale factor of 0.707 and our stopping criterion was a Bayes Factor $>10$ or $<0.1$. The scale of the default prior was chosen in accordance with plausible effect sizes in psychological research but the results presented are qualitatively unaffected by the exact choice. I set an upper maximum sample size of $\mathrm{n}=30$ but the stopping criterion was already reached at $\mathrm{n}=13$. In additional analyses, I also compared the biases between the various sub-conditions. Further, I conducted all of these analyses also for the discrimination ability (uncertainty). 
A
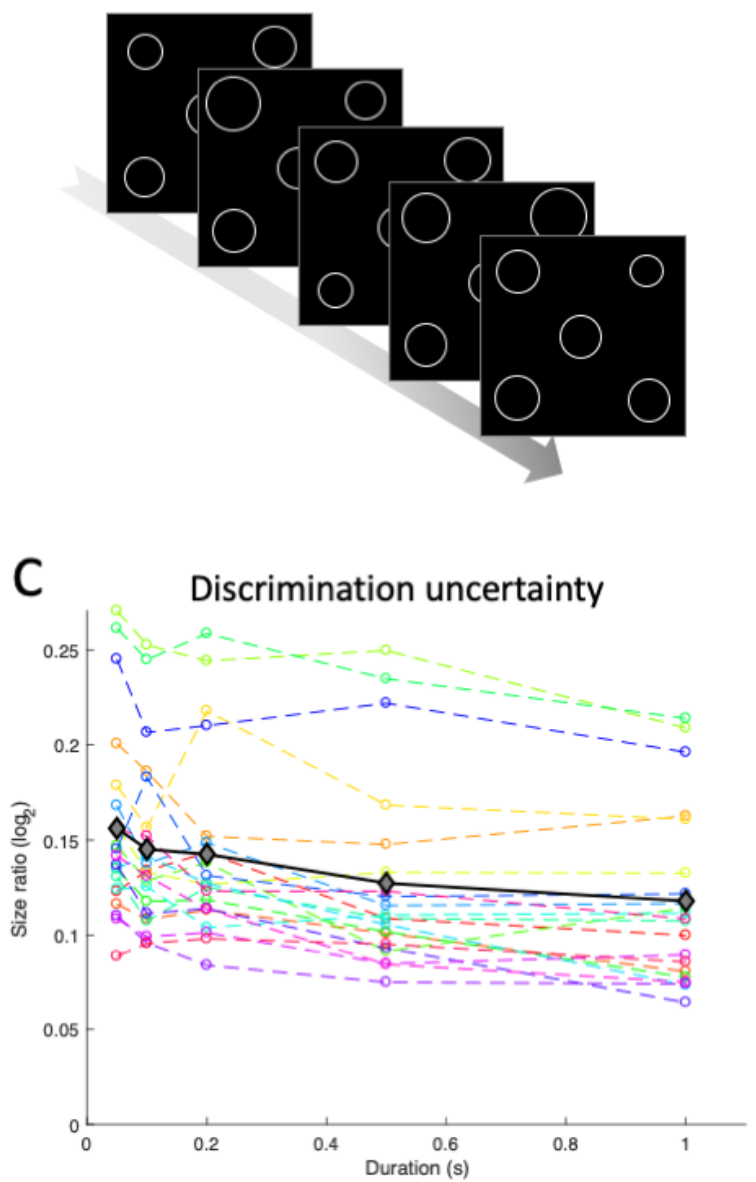

$\mathrm{B}_{0.25} \quad$ Perceptual bias

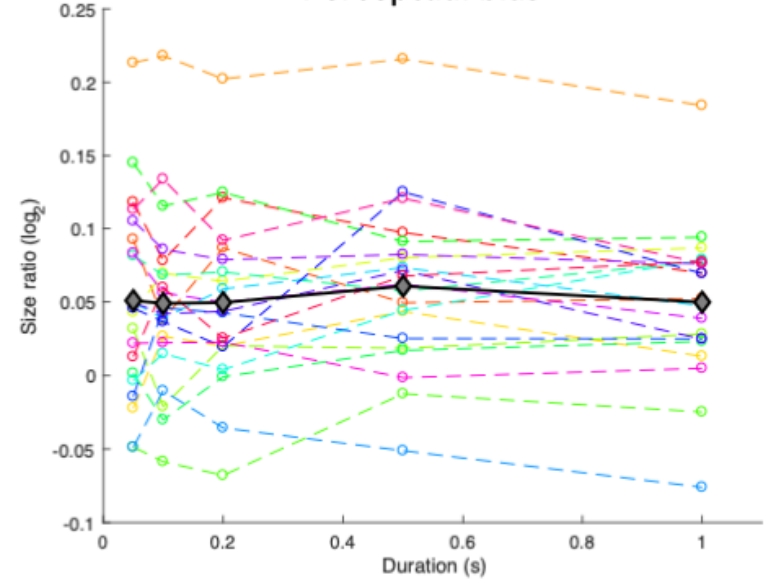

D

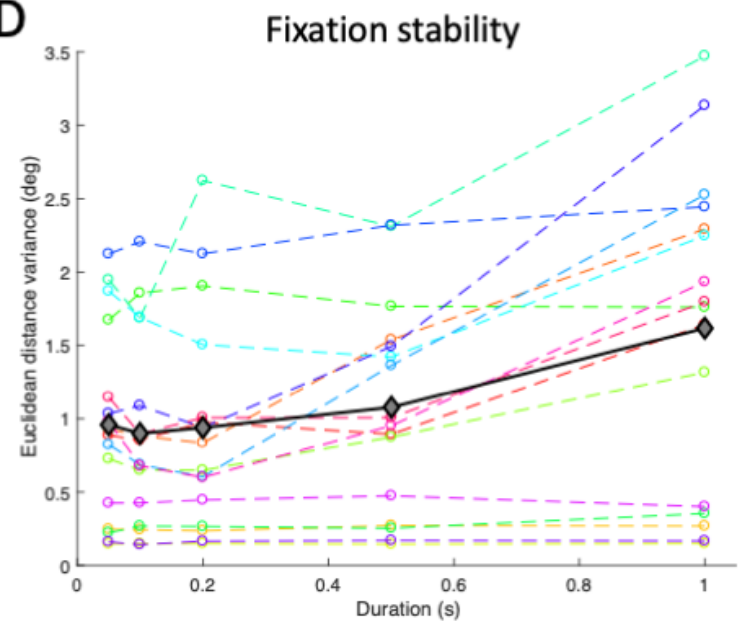

Figure 1. A. Schematic illustration of a sequence of trials in the MAPS task (Moutsiana et al., 2016) in Experiment 1. In each trial, observers were shown an array of circles and instructed to select the quadrant with the candidate circle that best matched the size of the central reference. B-D. Perceptual bias estimates (B), discrimination uncertainty (C), and fixation stability (D) plotted against stimulus duration. Dashed lines in colors denote individual observers. The solid black lines with diamond symbols denotes the group mean.

\section{Results}

\section{Experiment 1}

I used the MAPS task (Figure $1 \mathrm{~A}$ ) to test how perceptual biases and discrimination ability depended on stimulus duration. Mean bias estimates were stable regardless of stimulus duration (Figure 1B) with no statistically 
significant difference between different durations (1-way repeatedmeasures ANOVA: $F(4,80)=1.03, p=0.397)$. In contrast, discrimination ability, as quantified by the uncertainty parameter in MAPS (Figure 1C), was significantly better at longer stimulus durations $(F(4,80)=26.84$, $\mathrm{p}<0.001)$.

I further quantified the fixation stability across the trials. Separately for each duration condition, I calculated the deviation of eye movement positions in terms of their Euclidean distance (thus summarizing across horizontal and vertical eye position). This constitutes a measure of how far an observer's eye position varied across the experiment for each duration condition. Because eye tracking sometimes failed or produce artifactual large deviations ( $>4$ degrees of visual angle) the data from these participants and conditions were excluded. While a small number of observers maintained accurate fixation irrespective of stimulus duration, on average fixation stability decreased steadily and significantly $(F(4,60)=12.76, p<0.001)$ with longer stimulus duration (Figure 1D).

In summary, I found no effect of stimulus duration on the magnitude of perceptual biases. However, there was clear evidence of improved discrimination ability at longer stimulus duration while fixation stability was worse.

\section{Experiment 2}

I next tested if perceptual biases were stronger on the vertical than horizontal meridian (irrespective of eccentricity) as our model (Moutsiana et al., 2016) would predict based on a recent report that pRF spread is greater on the vertical meridian (Silva et al., 2017). Using a Bayesian sampling plan, I collected data until the Bayes Factor on a paired t-test comparing mean biases for the two meridians favored either the alternative or null hypothesis at a ratio of $10: 1$. The evidence clearly supported the alternative hypothesis $\left(t(12)=-3.61, p=0.004, B F_{10}=13.5\right)$, as the mean 
biases along the vertical meridian were almost twice as strong as those on the horizontal meridian (Figure $2 \mathrm{~A}$ ). Because a positive bias reflects how much a stimulus must be enlarged to be perceptually matched to the reference stimulus, the more positive the bias estimate the smaller the apparent stimulus size.

Separating data by eccentricity confirmed that this difference manifested both at $3.92^{\circ}$ eccentricity $\left(t(12)=-4.64, \mathrm{p}<0.001, \mathrm{BF}_{10}=64.2\right)$ and at $7.84^{\circ}$ eccentricity, although the latter effect was less consistent $(t(12)=-2.52$, $\left.\mathrm{p}=0.027, \mathrm{BF}_{10}=2.6\right)$. Finally, I also tested whether biases along the vertical meridian differed between the upper and lower hemifields (Figure 2B). Here I found no significant difference and statistical evidence instead weakly favored the null hypothesis $\left(t(12)=0.58, p=0.570, \mathrm{BF}_{10}=0.323\right)$.

A

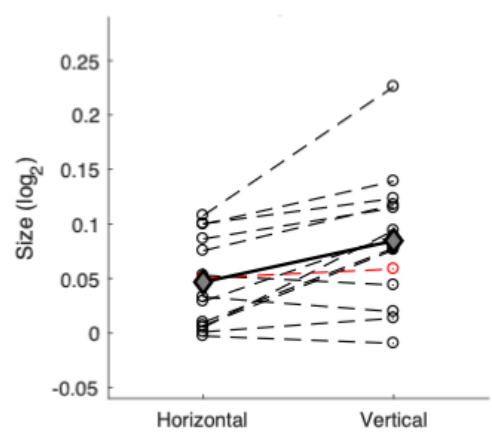

D

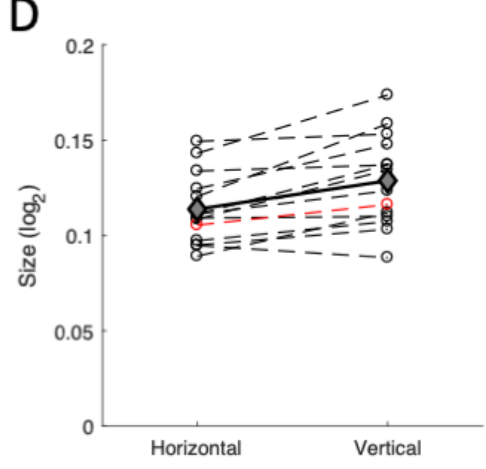

B

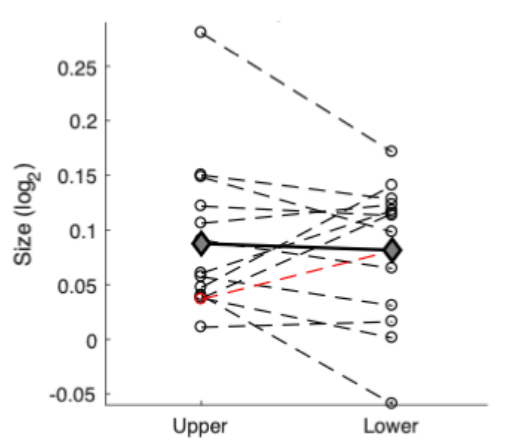

$\mathrm{E}$

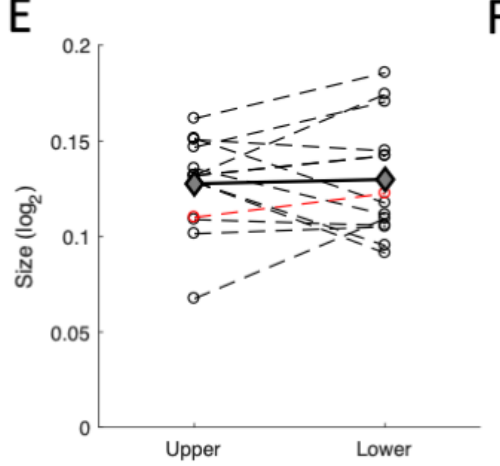

C

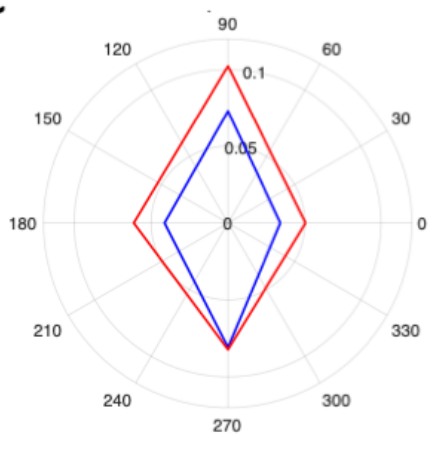

Eccentricity
$-3.92^{\circ}$

$\mathrm{F}$

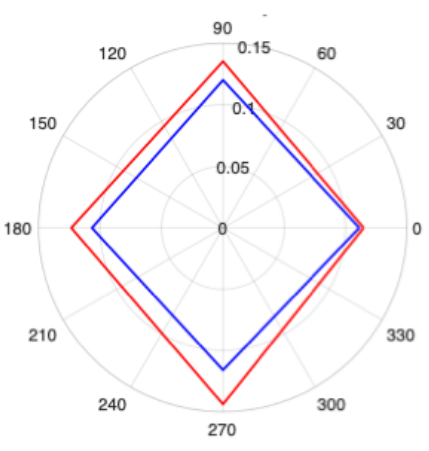

Figure 2. In Experiment 2, candidate stimuli were presented on the vertical and horizontal meridian (above, below, left, and right of the central reference). Perceptual bias (A-C) and discrimination uncertainty (D-F) estimates for the horizontal vs vertical meridian $(A, D)$, the upper vs lower visual field of the vertical meridian only $(B, E)$, and separately for each 
tested location $(C, F)$. Dashed lines in $A, B, C$, and $E$ denote individual observers. Solid black lines show the group mean. The red dashed line corresponds to the author's data which were excluded from statistical inference. In $C$ and $F$, data are separated by eccentricity (blue: $3.92^{\circ}$; red: $7.84^{\circ}$ ).

Next, I also conducted the same comparisons for the discrimination ability, as quantified by the uncertainty parameter in MAPS. Again, I found a significant difference between meridians (Figure 2D), with performance being better for the horizontal than the vertical meridian $(t(12)=-4.12$, $\left.\mathrm{p}=0.001, \mathrm{BF}_{10}=29.5\right)$. This difference was significant also specifically for the outer eccentricity of $7.84^{\circ}\left(t(12)=-4.69, p<0.001, B F_{10}=69.3\right)$ but not for the inner eccentricity of $3.92^{\circ}\left(t(12)=-1.59, p=0.137, B_{10}=0.766\right)$. As for perceptual biases, there was no significant difference in uncertainty between the upper and lower visual field on the vertical meridian (Figure $2 \mathrm{E})$ but rather results weakly favored the null hypothesis $(t(12)=-0.19$, $\left.\mathrm{p}=0.853, \mathrm{BF}_{10}=0.283\right)$.

The polar plots in Figure $2 \mathrm{C}$ and $2 \mathrm{~F}$ illustrate the mean perceptual biases and uncertainties across the group separately for each visual field position.

\section{Discussion}

In two experiments, I addressed questions about the spatial heterogeneity of size perception biases as measured by the MAPS task. Observers judged which of four candidate stimuli presented at parafoveal eccentricities best matched the size of a centrally presented reference. I then quantified the perceptual bias, at which size a candidate stimulus appeared the same as the reference, and the discrimination ability, how uncertain observers were in making their perceptual decisions. 
In our first experiment, I found that perceptual biases, a consistent underestimation of parafoveal stimulus size similar to those reported in previous studies, were constant irrespective of stimulus duration. Thus, even when observers have more time to view the stimuli, they still misperceive the size of stimuli. This finding is consistent with our model of how stimulus size is read out from their representation in visual cortex (Moutsiana et al., 2016).

Interestingly, discrimination ability, as quantified by the uncertainty parameter in MAPS, improved as stimulus duration increased. This result is unsurprising because it stands to reason that the task becomes easier when stimulus duration increases. Longer stimulus presentations mean that neuronal populations encoding the stimulus will fire for a longer time period. In turn, this means that the system can accumulate more data and thus permit more reliable estimates of its position and size.

It is also noteworthy that fixation stability became worse at longer stimulus durations. This is again unsurprising. A brief 50 ms stimulus presentation is far too short to allow any voluntary eye movements to be executed. In contrast, at our longest duration, 1000 ms, participants may have sometimes saccaded towards the parafoveal candidate stimuli and there would have been more opportunities for microsaccades and general eye movement jitter to occur. Worse fixation compliance for longer durations could also partly explain why discrimination ability increased at longer durations.

Critically, however, even though observers tended to make more eye movements during longer stimulus presentations, their perceptual biases remained unaffected. Theoretically, if observers actually fixated the individual stimuli in turn (which would still be challenging even at a duration of $1000 \mathrm{~ms}$ ) they should no longer have any perceptual bias. Yet even observers with relatively poor fixation compliance still largely maintained fixation and thus their perceptual biases were presumably unaffected by 
eye movements. These results are therefore consistent with our model linking the retinotopic responses to these stimuli to their apparent size.

In our second experiment, I then tested if perceptual biases were stronger along the vertical than the horizontal meridian. This was based on our previous model (Moutsiana et al., 2016) and the more recent finding that positional tuning, pRF spread, in early visual areas is broader along the vertical meridian (Silva et al., 2017). I confirmed this prediction in a preregistered design. Perceptual biases were indeed more pronounced, that is, stimuli were perceived as smaller when they were presented on the vertical than the horizontal meridian. This finding held for two different eccentricities tested. In addition, discrimination ability was also better along the horizontal meridian. This finding is also consistent with smaller pRFs along the horizontal meridian (Silva et al., 2017) and ties in with earlier reports of more accurate spatial vision on the horizontal meridian (Anderson et al., 2014; Carrasco et al., 2001).

In addition, I also conducted a secondary comparison of perceptual biases and discrimination ability between the upper and lower visual field. Previous work had suggested such hemifield differences for pRF spread (Silva et al., 2017); however, I found no evidence of such differences in terms of perception. This could be due to the fact that the earlier pRF differences between hemifields were far less pronounced than differences between the meridians. Naturally, I also had less data for this comparison because there were only half the number of measurements and therefore statistical power for this comparison is lower. There could also be considerable interindividual variability in the functional organization of the hemifields.

Taken together, our findings in this study show that perceptual biases for size judgements are temporally stable. Moreover, they are more pronounced along the vertical than the horizontal meridian, that is, stimuli appear smaller on the vertical meridian. These findings are consistent with our model of how the visual system infers object size from representations in early visual cortex. Responses are presumably sustained over the 
duration of stimulus presentation. While longer stimuli may improve discrimination performance because signals can be estimated more reliably, they do not become more accurate but remain distorted by the heterogeneity of positional tuning of cortical neurons.

\section{Data availability}

Data and materials are available for download (Experiment 1: osf.io/32jqu; Experiment 2: osf.io/au78z).

\section{Acknowledgements}

This work was supported by a ERC Starting Grant. I thank Samuel Spence for help with data collection.

\section{References}

Afraz, A., Pashkam, M. V., \& Cavanagh, P. (2010). Spatial heterogeneity in the perception of face and form attributes. Current Biology: $C B, 20(23), 2112-2116$. https://doi.org/10.1016/j.cub.2010.11.017

Anderson, J. E., Leslie Cameron, E., \& Levine, M. W. (2014). A method for quantifying visual field inhomogeneities. Vision Research, 105, 112-120. https://doi.org/10.1016/j.visres.2014.09.010

Anstis, S. (1998). Picturing peripheral acuity. Perception, 27(7), 817-825.

Carrasco, M., Talgar, C. P., \& Cameron, E. L. (2001). Characterizing visual performance fields: effects of transient covert attention, spatial frequency, eccentricity, task and set size. Spatial Vision, 15(1), 61-75.

Dumoulin, S. O., \& Wandell, B. A. (2008). Population receptive field estimates in human visual cortex. NeuroImage, 39(2), 647-660. https://doi.org/10.1016/j.neuroimage.2007.09.034 
Finlayson, N. J., Manser-Smith, K., Balraj, A., de Haas, B., \& Schwarzkopf, D. S. (2018). The optimal experimental design for multiple alternatives perceptual search. Attention, Perception, \& Psychophysics, 80(8), 1962-1973. https://doi.org/10.3758/s13414-018-1568-x

Finlayson, N. J., Papageorgiou, A., \& Schwarzkopf, D. S. (2017). A new method for mapping perceptual biases across visual space. Journal of Vision, 17(9), 5. https://doi.org/10.1167/17.9.5

Greenwood, J. A., Szinte, M., Sayim, B., \& Cavanagh, P. (2017). Variations in crowding, saccadic precision, and spatial localization reveal the shared topology of spatial vision. Proceedings of the National Academy of Sciences, 114(17), E3573-E3582. https://doi.org/10.1073/pnas.1615504114

Kosovicheva, A., \& Whitney, D. (2017). Stable individual signatures in object localization. Current Biology: CB, 27(14), R700-R701. https://doi.org/10.1016/j.cub.2017.06.001

Moutsiana, C., de Haas, B., Papageorgiou, A., van Dijk, J. A., Balraj, A., Greenwood, J. A., \& Schwarzkopf, D. S. (2016). Cortical idiosyncrasies predict the perception of object size. Nature Communications, 7, 12110. https://doi.org/10.1038/ncomms12110

Newsome, L. R. (1972). Visual angle and apparent size of objects in peripheral vision. Perception \& Psychophysics, 12(3), 300-304. https://doi.org/10.3758/BF03207209

Rouder, J. N., Speckman, P. L., Sun, D., Morey, R. D., \& Iverson, G. (2009). Bayesian t tests for accepting and rejecting the null hypothesis. Psychonomic Bulletin \& Review, 16(2), 225-237. https://doi.org/10.3758/PBR.16.2.225

Schwarzkopf, D. S., \& Rees, G. (2013). Subjective size perception depends on central visual cortical magnification in human v1. PloS One, 8(3), e60550. https://doi.org/10.1371/journal.pone.0060550

Silva, M. F., Brascamp, J. W., Ferreira, S., Castelo-Branco, M., Dumoulin, S. O., \& Harvey, B. M. (2017). Radial asymmetries in population receptive field size and 
cortical magnification factor in early visual cortex. NeuroImage, 167, 41-52. https://doi.org/10.1016/j.neuroimage.2017.11.021

Smith, A. T., Singh, K. D., Williams, A. L., \& Greenlee, M. W. (2001). Estimating receptive field size from fMRI data in human striate and extrastriate visual cortex. Cerebral Cortex (New York, N.Y.: 1991), 11(12), 1182-1190.

Visconti di Oleggio Castello, M., Taylor, M., Cavanagh, P., \& Gobbini, M. I. (2018). Idiosyncratic, Retinotopic Bias in Face Identification Modulated by Familiarity. ENeuro, 5(5). https://doi.org/10.1523/ENEURO.0054-18.2018 